\title{
Pilot in vivo toxicological investigation of boron nitride nanotubes
}

This article was published in the following Dove Press journal:

International Journal of Nanomedicine

30 December 201I

Number of times this article has been viewed

\author{
Gianni Ciofani' \\ Serena Danti ${ }^{2}$ \\ Giada Graziana Genchi ${ }^{1,3}$ \\ Delfo D'Alessandro² \\ Jean-Luc Pellequer ${ }^{4}$ \\ Michaël Odorico ${ }^{4}$ \\ Virgilio Mattoli' \\ Mario Giorgi ${ }^{5}$ \\ 'Italian Institute of Technology, Center \\ of MicroBioRobotics clo Scuola \\ Superiore Sant'Anna, ${ }^{2}$ Department \\ of Neuroscience, University of Pisa, \\ ${ }^{3}$ The BioRobotics Institute, Scuola \\ Superiore Sant'Anna, Pisa, Italy; \\ ${ }^{4}$ Commissariat à l'Energie Atomique, \\ Institut de Biologie Environnementale \\ et Biotechnologie, Department of \\ Biochemistry and Nuclear Toxicology, \\ Bagnols-sur-Cèze, France; ${ }^{5}$ Division \\ of Pharmacology and Toxicology, \\ Veterinary Clinics Department, \\ University of Pisa, Pisa, Italy
}

Correspondence: Gianni Ciofani Italian Institute of Technology, Center for Micro-BioRobotics c/o Scuola Superiore Sant'Anna, Viale Rinaldo Piaggio,

34-56025 Pontedera (Pisa), Italy

Tel +39050883019

Fax +39050 883497

Email g.ciofani@sssup.it

\begin{abstract}
Boron nitride nanotubes (BNNTs) have attracted huge attention in many different research fields thanks to their outstanding chemical and physical properties. During recent years, our group has pioneered the use of BNNTs for biomedical applications, first of all assessing their in vitro cytocompatibility on many different cell lines. At this point, in vivo investigations are necessary before proceeding toward realistic developments of the proposed applications. In this communication, we report a pilot toxicological study of BNNTs in rabbits. Animals were injected with a $1 \mathrm{mg} / \mathrm{kg}$ BNNT solution and blood tests were performed up to 72 hours after injection. The analyses aimed at evaluating any acute alteration of hematic parameters that could represent evidence of functional impairment in blood, liver, and kidneys. Even if preliminary, the data are highly promising, as they showed no adverse effects on all the evaluated parameters, and therefore suggest the possibility of the realistic application of BNNTs in the biomedical field.
\end{abstract}

Keywords: boron nitride nanotubes, in vivo testing, toxicology

\section{Introduction}

Boron nitride nanotubes (BNNTs), similar to carbon nanotubes (CNTs), have attracted wide attention thanks to their potentially unique and important properties in structural and electronic applications. ${ }^{1,2}$ A BNNT is a structural analog of a CNT, but, despite this similarity, it presents many different chemical and physical properties. For instance, BNNTs display excellent mechanical properties, with a measured Young's modulus of 1.22 $\pm 0.24 \mathrm{TPa}$. Moreover, while CNTs exhibit either a semiconductive or conductive behavior depending on chirality and diameter, BNNTs have only a constant band gap of about $5.5 \mathrm{eV}^{3} \mathrm{Ab}$ initio calculations ${ }^{4}$ and experimental data, ${ }^{5}$ moreover, demonstrated that they work as excellent piezoelectric systems, with response values larger than those of piezoelectric polymers and comparable to those exhibited by wurtzite semiconductors.

BNNTs have also attracted attention in the fields of nanomedicine, both as nanovectors for drug delivery purposes ${ }^{6}$ and as intracellular nanotransducers. ${ }^{7}$ Data on cytocompatibility of these innovative nanovectors come from studies of BNNTs incubated with different cell types, like human embryonic kidney cells, HEK 293; Chinese hamster ovary cells, $\mathrm{CHO} ;{ }^{8}$ human osteoblasts or mouse macrophages; ${ }^{9}$ human neuroblastoma cells, SH-SY5Y; ${ }^{10}$ mouse myoblasts, $\mathrm{C} 2 \mathrm{C} 12 ;{ }^{11}$ and neuronallike PC12 cells. ${ }^{7}$

Results reported by many groups strongly agree about the optimal cytocompatibility of BNNTs, as their administration did not affect cell proliferation, metabolism, 
and differentiation. However, a recent report by Horvath and coworkers outlined possible adverse effects of BNNTs on fibroblasts, macrophages, and lung cells. ${ }^{12}$ A possible explanation for such different results may deal with the considerable length (tens of microns) of the tested BNNTs: the toxicity of nanomaterials is, in fact, well-known as being connected to the aspect ratio of tubular nanostructures. ${ }^{13}$

At this point, as extensive cytocompatibility investigations of BNNTs have been performed, in vivo testing (ie, biocompatibility studies) is fundamental to answer open questions and direct scientific efforts toward realistic applications of BNNTs in the biomedical fields. Here, we report the first toxicology investigation of BNNTs in animals. Although preliminary, our results suggest a good response of the subjects to intravenous injections of BNNT dispersions, with no adverse effects on blood cells, liver, and kidneys.

\section{Materials and methods Preparation of BNNT dispersions}

BNNTs were purchased from the Nano and Ceramic Materials Research Center, Wuhan Institute of Technology, China. ${ }^{14}$ Details about production and characterization of the samples, and about the preparation of the dispersions, can be found elsewhere. ${ }^{10}$ Briefly, glycol chitosan (G-chitosan, G7753; Sigma-Aldrich Co, St Louis, MO) was used for the stabilization of BNNTs in aqueous medium, and nanotube noncovalent coating was achieved through sonication at $20 \mathrm{~W}$ for 12 hours with a Bransonic Sonicator 2510 (Danbury, CT). In this study, dispersions were prepared in physiological solution $(0.9 \% \mathrm{NaCl}$; Eurospital, Trieste, Italy) by mixing polymer and BNNTs at a 1:1 (w/w) ratio, thus achieving a final concentration of $1 \mathrm{mg} / \mathrm{mL}$ BNNTs, coated with $1 \mathrm{mg}$ / $\mathrm{mL}$ of G-chitosan. Before injection, dispersions were autoclaved for sterilization.

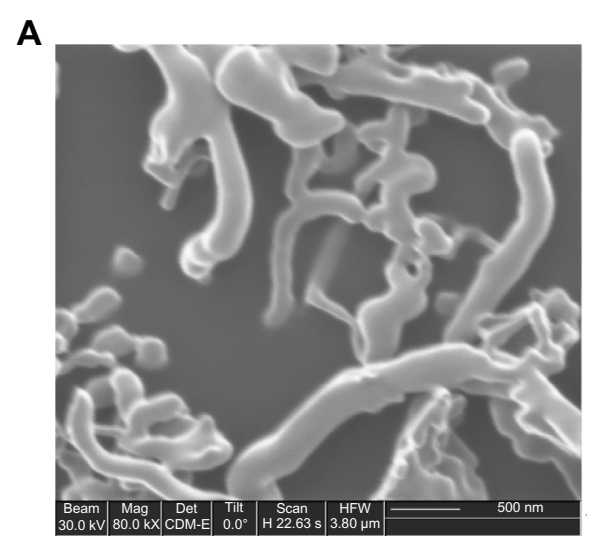

\section{Characterization of BNNT dispersions}

Microphotographs of the final dispersions of BNNTs were obtained through focused-ion beam microscopy (FIB) with a FEI 200 microscope (FEI HQ, Hillsboro, OR). Transmission electron microscopy (TEM) was performed with a Zeiss 902 TEM (Carl Zeiss, GmbH, Germany) dropping a small quantity of BNNT aqueous suspension (diluted 1000×) on a copper grid. For atomic force microscopy (AFM) imaging, $50 \mu \mathrm{L}$ of BNNT solution (diluted $2500 \times$ ) was deposited on freshly cleaved, highly ordered pyrolitic graphite (HOPG; Bruker AXS, Inc, Madison, WI). Graphite substrates were incubated for 6 minutes and rinsed five times with $100 \mu \mathrm{L}$ of pure water. Samples were then vacuum dried using a Laboport vacuum pump (KNF Neuberger, Inc, Trenton, NJ). Imaging was performed on a Multimode scanner with a Nanoscope V controller, using the Scan-Assyst mode (Bruker AXS). Imaging scan rate was $1.95 \mathrm{~Hz}$ for a scan size of $250 \mathrm{~nm}$. Images were recorded at $512 \times 512$ pixels and stripe noise was evaluated using the DeStripe server. ${ }^{15}$ V-shaped silicon nitride cantilevers with silicon tips (SNL; Bruker AXS) with a nominal spring constant of $0.35 \mathrm{~N} / \mathrm{m}$ were used. Imaging forces were maintained below $100 \mathrm{pN}$.

Particle size distribution and Z-potential of the dispersions were analyzed with a Nano Z-Sizer 90 (Malvern Instruments Ltd, Malvern, UK). For both analyses, each acquisition was performed three times, using samples appropriately diluted in physiological solution.

\section{Animal treatment}

Five male New Zealand rabbits, aged 8-9 months, and weighing $2.0 \pm 0.1 \mathrm{~kg}$, were used in the present study. Animal care and handling were performed according to the provisions of Council Directive 86/609 EEC, recognised and adopted by the Italian Government (DL 27/1/1992, n 16). The experimental

B

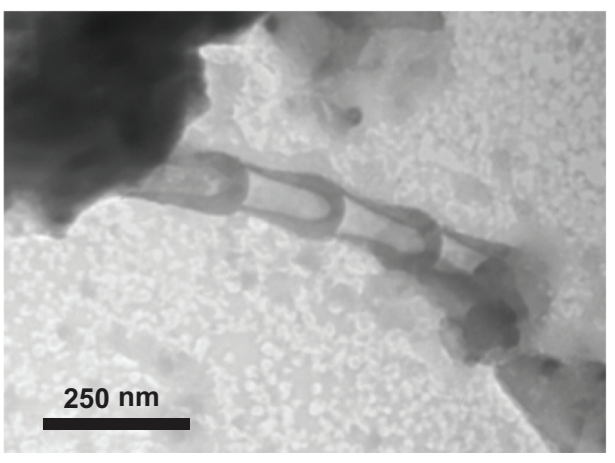

Figure I (A) Focused-ion beam microscopy image of G-chitosan-coated boron nitride nanotubes (BNNTs) and (B) transmission electron microscopy analysis showing the typical BNNT structure. 

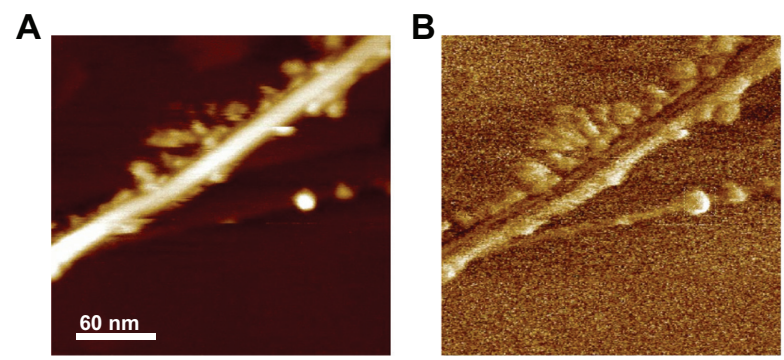

Figure 2 (A) Height and (B) phase atomic force microscopy images of G-chitosan coated boron nitride nanotubes.

protocol was approved by the Ethics Committee of the University of Pisa and by the Italian Ministry of Health (authorization n 4886, 04/04/2011). Animal health was clinically assessed through physical examination and complete hematological analyses before experimental sessions.

Rabbits were housed in single cages, under conventional conditions of ventilation, temperature $\left(18^{\circ} \mathrm{C}-22^{\circ} \mathrm{C}\right)$ and lighting (16-hour light:day cycle). Animals were allowed to adjust to their environment for 1 week. Food and water were available ad libitum during the whole trial. Animals had a diet of complete premixed food for rabbits (Consorzio Agrario della Maremma Toscana, Grosseto, Italy) and alfalfa hay. For the whole experimental period, general rabbit health was daily monitored by qualified personnel supervised by a veterinary physician.

Animals were randomly assigned to two groups: a control group (I, $n=2$ ) and an experimental group (II, $n=3$ ). Each subject in group I and group II received a single intravenous dose $(1 \mathrm{mg} / \mathrm{kg})$ of plain G-chitosan and G-chitosan-coated BNNTs in the morning. The two solutions, having a concentration of $1 \mathrm{mg} / \mathrm{mL}$, allowed for injections of about $2 \mathrm{~mL}$ volume. Solutions were slowly injected into the marginal ear vein of animals placed in restraining cages. Objective symptoms such as sweating, excitement, trembling, and head nodding were scored. Body temperature was also assessed during the study. Blood samples were collected for analysis at intervals of $0,2,24$ and 72 hours by the contralateral marginal ear vein used for administration, and they were placed in collection tubes containing appropriate anticoagulants. Blood samples were processed and analyzed at the Biochemistry Clinical Veterinary Laboratory of the Veterinary Teaching Hospital, Pisa, Italy, within 2 hours of collection.

\section{Results and discussion}

BNNTs were produced by using an annealing method from boron-containing precursors. Details of sample, provided by

A

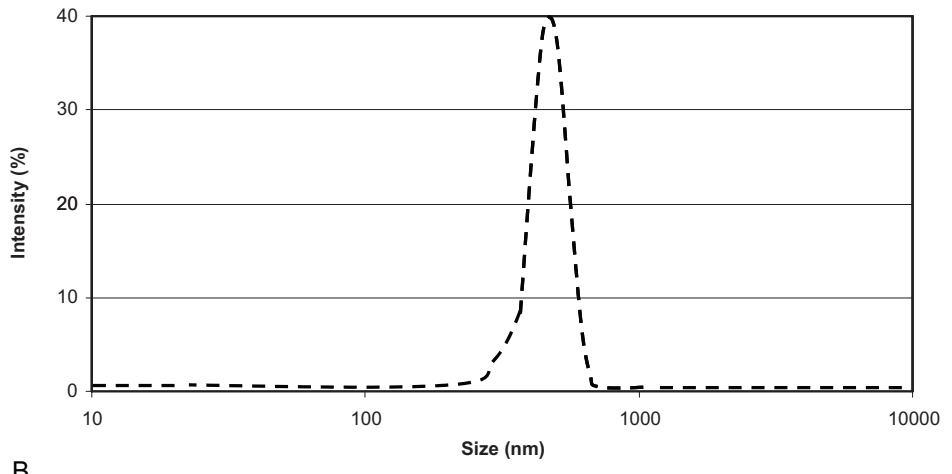

B

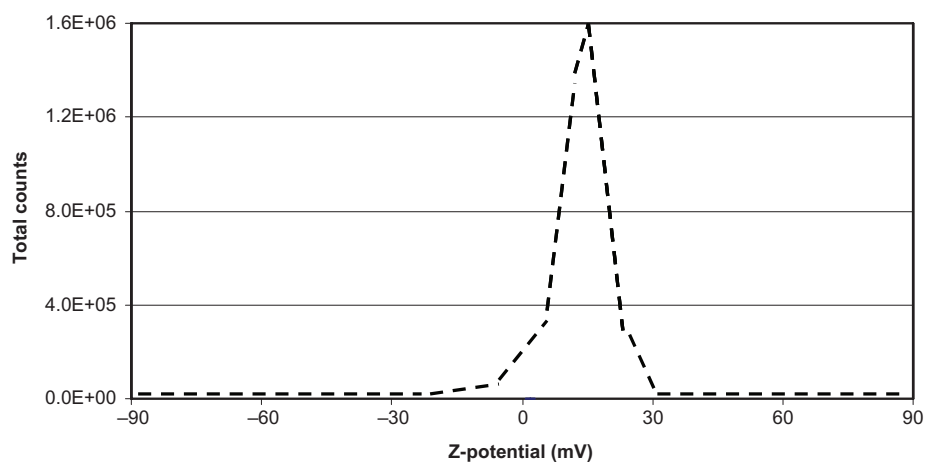

Figure 3 (A) Size distribution and (B) Z-potential analyses of the injected boron nitride nanotube dispersions. 


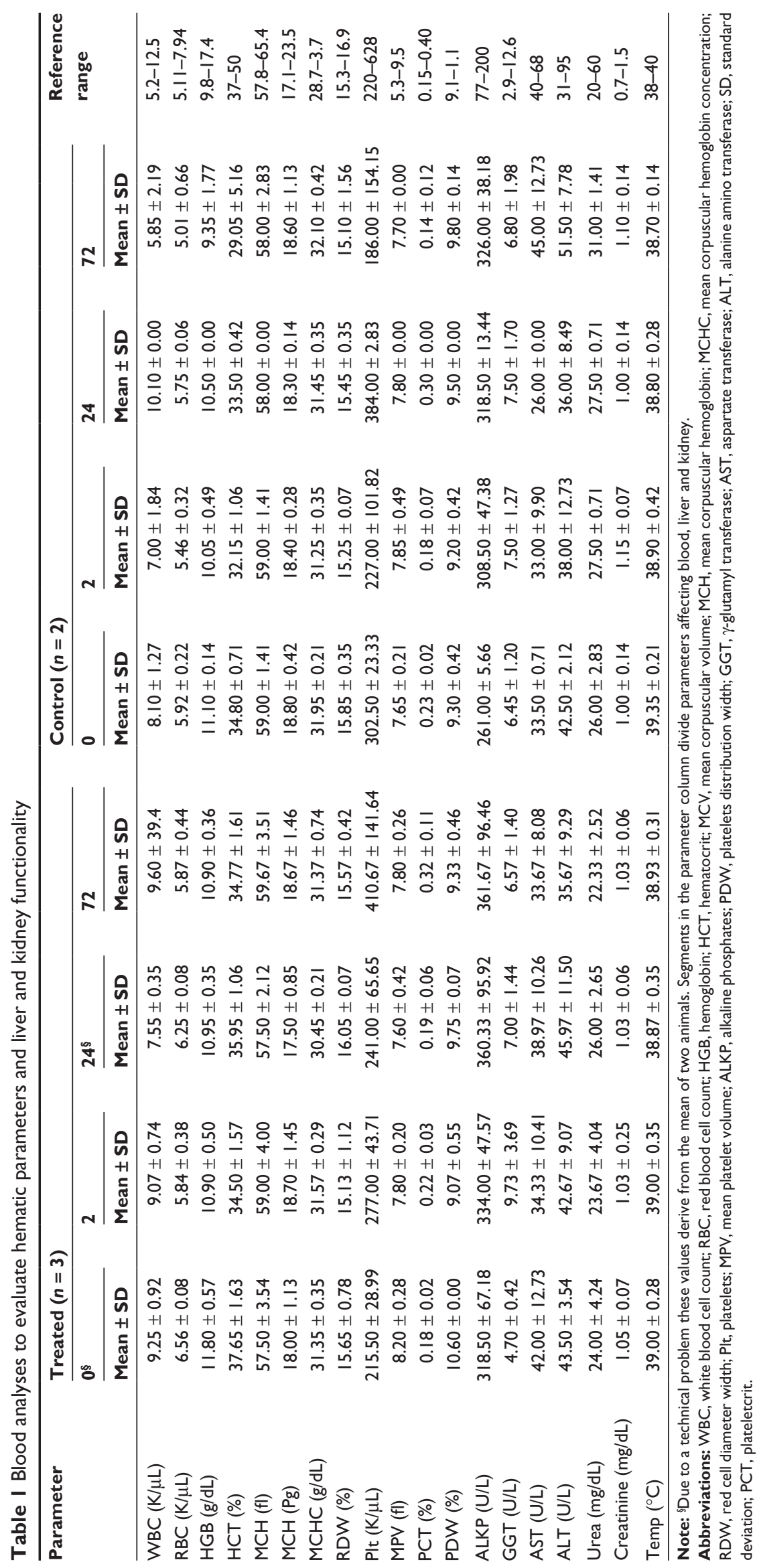


the supplier included a boron nitride content $>98.5 \mathrm{wt} \%$ and yield of the procedure $>80 \%$. A more detailed characterization of BNNTs and of their dispersions was reported in a previous work. ${ }^{10}$

Figure 1 shows the results of FIB and TEM imaging on the G-chitosan-coated BNNTs used in animal experiments. The presence of tubular structures with random orientation and quite dispersed size distribution (tube diameters were between $30 \mathrm{~nm}$ and $100 \mathrm{~nm}$ and lengths between $0.5 \mu \mathrm{m}$ and $2.0 \mu \mathrm{m}$ ) can be seen in the FIB image (Figure 1A), whereas the typical bamboo-like structure of BNNTs, ${ }^{16}$ associated with a thin G-chitosan coating (around the BNNT walls), can be appreciated in the TEM image (Figure 1B).

AFM imaging shows an isolated nanotube: in the height image (Figure 2A), the nanotube appears as a bright line with edges decorated by globular structures, whereas it appears as a dark line decorated by brighter globular structures in the phase image (Figure 2B). The latter once more confirms the efficiency of G-chitosan-coating procedure: the phase delay introduced by the globular structures, which can be appreciated through the brighter color, is due to the G-chitosan lower stiffness with respect to that of BNNTs, which in fact display a darker color. ${ }^{17}$

To characterize BNNT assembling in aqueous medium and to gain an idea of the actual sizes of the nanocomplexes in the injected dispersions (FIB, TEM, and AFM analyses were carried out on dried samples), dynamic light scattering and Z-potential analyses were performed in physiological solution. Dynamic light scattering revealed a $96.5 \%$ peak at a size of about $560 \mathrm{~nm}$, with a polydispersion index of 0.250 (Figure 3A). Moreover, a Z-potential of about $15 \mathrm{mV}$ denoted a good stability of the obtained dispersion (Figure 3B).

During the whole study period, neither unusual behavior, such as sweating, excitement, trembling, and head nodding, nor differences could be observed between the two groups. Moreover, no significant changes in body temperature were observed up to 72 hours following the injection.

Results on hematological analyses are reported in detail in Table 1. Typical blood values (white cell count, red cell count, platelet count, etc) are not significantly different between control (I, ie, treated with G-chitosan) and experimental (II, ie, treated with G-chitosan-coated BNNTs) groups. Only platelet count seems to be increased at 72 hours after injection in the BNNT-treated group, but this value is still in the healthy range tabulated for rabbits. ${ }^{18}$

Blood biochemical parameters quantifying both renal (urea and creatinine) and hepatic (alkaline phosphatase, $\gamma$-glutamyl transferase, aspartate transferase, and alanine amino transferase) functions were also satisfactory: no substantial differences can be appreciated between the two groups at each time-point analysis.

\section{Conclusion}

This study demonstrates the first in vivo toxicity evaluation of BNNTs following intravenous injection. Single administrations of high doses did not produce evident toxicity in rabbits within the 72 hours following injection. Because of the small number of animals, our findings must be considered preliminary; nonetheless, the absence of negative effects on blood parameters, as well as liver and kidney functions is highly promising for further biocompatibility exploration of BNNTs. Future investigations will consist of dose-response and long-term studies. Particular attention will be also given to the investigation of the BNNT biodistribution and pharmacokinetics that are strictly dependent on the physicochemical characteristics of the nanoparticles, such as size, shape, aggregation, chemical composition, surface functionalizations, and solubility. ${ }^{19}$

\section{Acknowledgments}

AFM imaging was performed during the 3rd International AFMBioMed summer school in Marcoule in cooperation with the European COST Action TD1002 (Chair: Dr Pierre Parot). The authors thank Dr Matilde Masini, Department of Experimental Pathology BMIE, University of Pisa, Pisa, Italy, for TEM technical support.

\section{Disclosure}

The authors report no conflicts of interest in this work.

\section{References}

1. Chopra NG, Luyken RJ, Cherrey K, et al. Boron nitride nanotubes. Science. 1995;269(5226):966-967.

2. Terrones M, Romo-Herrera JM, Cruz-Silva E, et al. Pure and doped boron nitride nanotubes. Materials Today. 2007;10:30-38.

3. Golberg D, Bando Y, Tang C, Zhi C. Boron nitride nanotubes. Adv Mater. 2007;19:2413-2432.

4. Dai Y, Guo W, Zhang Z, Zhou B, Tang C. Electric-field-induced deformation in boron nitride nanotubes. J Phys D Appl Phys. 2009;42:085403.

5. Bai XD, Golberg D, Bando Y, Zhi CY, Tang C, Mitome M. Deformationdriven electrical transport of individual boron nitride nanotubes. Nano Lett. 2007;7(3):632-637.

6. Ciofani G. Potential applications of boron nitride nanotubes as drug delivery systems. Expert Opin Drug Deliv. 2010;7(8):889-893.

7. Ciofani G, Danti S, D'Alessandro D, et al. Enhancement of neurite outgrowth in neuronal-like cells following boron nitride nanotube-mediated stimulation. ACS Nano. 2010;4(10):6267-6277.

8. Chen X, Wu P, Rousseas M, et al. Boron nitride nanotubes are noncytotoxic and can be functionalized for interaction with proteins and cells. J Am Chem Soc. 2009;131:890-891. 
9. Lahiri D, Rouzaud F, Richard T, et al. Boron nitride nanotube reinforced polylactide-polycaprolactone copolymer composite: mechanical properties and cytocompatibility with osteoblasts and macrophages in vitro. Acta Biomater. 2010;6(9):3524-3533.

10. Ciofani G, Danti S, D’Alessandro D, Moscato S, Menciassi A. Assessing cytotoxicity of boron nitride nanotubes: Interference with the MTT assay. Biochem Biophys Res Commun. 2010;394(2):405-411.

11. Ciofani G, Ricotti L, Danti S, et al. Investigation of interactions between poly-L-lysine-coated boron nitride nanotubes and $\mathrm{C} 2 \mathrm{C} 12$ cells: up-take, cytocompatibility, and differentiation. Int J Nanomedicine. 2010;5:285-298.

12. Horváth L, Magrez A, Golberg D, et al. In vitro investigation of the cellular toxicity of boron nitride nanotubes. ACS Nano. 2011;5(5):3800-3810.

13. Poland CA, Duffin R, Kinloch I, et al. Carbon nanotubes introduced into the abdominal cavity of mice show asbestos-like pathogenicity in a pilot study. Nat Nanotechnol. 2008;3:423-428.
14. Wang J, Gu Y, Zhang L, Zhao G, Zhang Z. Synthesis of boron nitride nanotubes by self-propagation high-temperature synthesis and annealing method. J Nanomater. 2010; article ID:540456.

15. Chen SW, Pellequer JL. DeStripe: frequency-based algorithm for removing stripe noises from AFM images. BMC Struct Biol. 2011;11:7.

16. Tang CC, Lamy de la Chapelle M, Li P, Liu YM, Dang HY, Fan SS. Catalytic growth of nanotube and nanobamboo structures of boron nitride. Chem Phys Lett. 2001;342(5-6):492-496.

17. Jandt K. Atomic force microscopy of biomaterials surfaces and interfaces. Surface Science. 2001;491:303-332.

18. Moore DM. Hematology of rabbits and hematology of the guinea pig. In: Feldman BF, Zinkl JG, Jain NC, editors. Schalm's Veterinary Hematology. 5th ed. Baltimore, MD: Lippincott Williams \& Wilkins; 2000:1100-1110.

19. Lacerda L, Bianco A, Prato M, Kostarelos K. Carbon nanotubes as nanomedicines: from toxicology to pharmacology. Adv Drug Deliv Rev. 2006;58(14):1460-1470.
International Journal of Nanomedicine

\section{Publish your work in this journal}

The International Journal of Nanomedicine is an international, peerreviewed journal focusing on the application of nanotechnology in diagnostics, therapeutics, and drug delivery systems throughout the biomedical field. This journal is indexed on PubMed Central, MedLine, CAS, SciSearch ${ }^{\circledR}$, Current Contents ${ }^{\circledR} /$ Clinical Medicine,

\section{Dovepress}

Journal Citation Reports/Science Edition, EMBase, Scopus and the Elsevier Bibliographic databases. The manuscript management system is completely online and includes a very quick and fair peer-review system, which is all easy to use. Visit http://www.dovepress.com/ testimonials.php to read real quotes from published authors. 